\title{
Bem-estar da puérpera no atendimento ao parto em uma maternidade municipal no norte do Brasil
}

\author{
Well-being of puerperal women during childbirth care in a municipal maternity hospital in \\ northern Brazil
}
Bienestar de las puérperas durante la atención al parto en una maternidad municipal del norte de Brasil

Olivanda de Oliveira ${ }^{1}$, Roberta Claudinha Reges ${ }^{1 *}$, Silvana Capiche ${ }^{1}$.

\begin{abstract}
RESUMO
Objetivo: Analisar os fatores relacionados ao bem-estar no parto na perspectiva de mulheres atendidas em uma maternidade no norte do Brasil. Métodos: Trata-se de uma pesquisa exploratória de abordagem quantitativa. O estudo se propõe a utilizar a escala de Bem-Estar Materno em Situação de Parto (BMSP) que aborda o bem-estar da mulher em situação de parto de maneira multidimensional. Resultados: Participaram deste estudo 56 puérperas. Obteve-se um total de pontos da escala de 198 média e 204,5 mediana, o local de estudo classifica-se em adequado. Dos sete domínios avaliados, o único domínio com valor abaixo do ponto de corte refere-se ao item "Cuidado despersonalizado", com $72 \%$, tal domínio avalia ao relacionamento interpessoal estabelecido pelos profissionais. Conclusão: $O$ estudo apresentou dados que possibilitaram a reflexão a respeito da assistência. Os principais resultados mostram que o bem-estar materno está presente na unidade estudada, apesar do ponto relacionado ao cuidado despersonalizado requerer investimento.
\end{abstract}

Palavras-chave: Bem-estar, Parto humanizado, Maternidade, Boas práticas no parto.

\begin{abstract}
Objective: To analyze the factors related to well-being in childbirth from the perspective of women assisted in a maternity hospital in northern Brazil. Methods: This is an exploratory research with a quantitative approach. O estudo se propõe a utilizar a escala de Bem-Estarstar Materno em Situação de parto (BMSP) que aborda o bem-estar da mulher em situação de parto de maneira multidimensional Resultados: Participaram deste estudo 56 puérperas. We obtained a total scale score of 198 mean and 204.5 median, the study site is classified as adequate. Of the seven domains assessed, the only domain with a value below the cutoff point was the item "Depersonalized care", with $72 \%$ this domain assesses the interpersonal relationship established by professionals. Conclusion: The study presented data that allowed reflection about the assistance. The main results show that maternal well-being is present in the unit studied, although the point related to depersonalized care requires investment.
\end{abstract}

Palavras-chave: Well-being, Humanizing childbirth, Maternity, Good practices in childbirth.

\section{RESUMEN}

Objetivo: Analizar los factores relacionados con el bienestar en el parto desde la perspectiva de las mujeres atendidas en una maternidad del norte de Brasil. Métodos: Se trata de una investigación exploratoria con un enfoque cuantitativo. El estudio se propone utilizar la escala de Bem-Estar Materno en Situación de Parto (BMSP) que aborda el bienestar de la mujer en situación de parto de manera multidimensional. Resultados:

${ }^{1}$ Faculdade Interamericana de Porto Velho - UNIRON, Porto Velho - RO.

*E-mail: roberta_reges@hotmail.com 
Participaron en este estudio 56 puérperas. Al obtener una puntuación total en la escala de 198 de media y 204,5 de mediana, el lugar del estudio se clasifica como adecuado. De los siete dominios evaluados, el único dominio con valor por encima del punto de corte se refiere al ítem "Cuidado despersonalizado", con un 72\%, dicho dominio se refiere a la relación interpersonal establecida por los profesionales. Conclusión: El estudio presentó datos que permitieron reflexionar sobre la asistencia. Los principales resultados muestran que el bienestar materno está presente en la unidad estudiada, aunque el punto relacionado con la atención despersonalizada requiere inversión.

Palavras-chave: Bienestar, Nacimiento humanizado, Maternidad, Buenas prácticas en el parto.

\section{INTRODUÇÃO}

O parto humanizado pode ser descrito como um conjunto de condutas e procedimentos que têm por finalidade a promoção do parto e nascimento saudáveis e a prevenção da morbimortalidade materna e Perinatal. Ou seja, pode ser definido como um processo que respeita a individualidade da mulher, valorizando-a como protagonista e permitindo a adequação da assistência a cultura, crenças, valores e diversidade de opinião dessas pessoas (TORAL A, et al., 2019).

A nova posição da mulher no processo do parto humanizado, trouxeram mudanças importantes, a inovação está tanto na capacidade dessas mulheres de exercer autonomia, como de escolher um acompanhante, todos os procedimentos voltados para essa assistência sejam muito bem esclarecidos á elas e ao acompanhante (LIMA PO, et al., 2018).

O Programa de Humanização do Pré-Ntatal e Nascimento (PHPN) trouxe muitas atualizações e recomendações nas práticas clínicas que já existiam na época, transformando o processo de parto em um ambiente de acolhimento, indo desde o pré-natal até o nascimento. Dentre as variáveis que o programa discute, temos, inserção de um acompanhante de livre escolha da mulher, qualificação das relações interpessoais entre profissionais e parturientes, produção de espaços de construção de saberes e informações, a participação, autonomia e controle decisório da mulher sobre o seu corpo etc (PEREIRA RM, et al., 2018).

A humanização da assistência é de extrema importância, pois garante que o parto seja um momento único, vivenciado de forma positiva e enriquecedora. Resgatando o contato humano, ouvindo, acolhendo, explicando, criando vínculo, todos são quesitos indispensáveis no cuidado. A partir disso a assistência da parturiente se tornou evidente e vem sendo discutida, de forma a tornar o processo de parir e nascer um espaço para a promoção à saúde da mulher (FERREIRA RC, et al., 2021; CAMPOS VZ, et al., 2020).

Os profissionais de saúde são coadjuvantes dessas experiências desempenhando um papel que ele coloque o seu conhecimento científico, de forma a proporcionar o bem-estar a mãe e ao bebê, tornando o parto inserido em um ambiente saudável. Ao prestar uma assistência de qualidade a puérpera, os profissionais de saúde têm um papel importante, onde podem ajudá-la a superar medos, tensões e ansiedade, com empatia e respeito, respeitando suas necessidades (ROCHA NFF e FERREIRA J, 2020).

Jamas MT (2013), após realizar busca em base de dados e verificar que no Brasil não existia escala validada para mensurar a satisfação ou o bem-estar das mulheres no momento da parturição, realizou a adaptação e tradução para a língua portuguesa (Brasil) do instrumento denominado "Escala de Bienestar Materno em Situación de Parto 2", além disso concluiu em seu estudo que deveriam ser realizados maiores investigações com diversas mulheres em outros estados do Brasil (BMSP 2).

Nessa perspectiva, faz-se relevante a pesquisa, visto que poderá servir de subsídio para fundamentar intervenções que busquem contribuir com ações relacionadas a assistência a mulher no parto e minimizar os fatores que causam desconfortos neste momento de grande importância para a mulher. $\mathrm{O}$ objetivo do estudo foi analisar os fatores relacionados ao bem-estar no parto na perspectiva de mulheres atendidas em uma maternidade municipal no norte do Brasil e medir a percepção de bem-estar materno. 


\section{MÉTODOS}

Trata-se de uma pesquisa exploratório de abordagem quantitativa que propõe a análise dos fatores relacionados ao bem-estar em uma maternidade municipal no norte do Brasil na percepção das mulheres.

Neste sentido, o estudo se propõe a utilizar a escala de Bem-Estar Materno em Situação de Parto (BMSP 2) que aborda o bem-estar da mulher em situação de parto, a escala é autoaplicável e tendo uma duração média de 20 minutos. A mesma busca mensurar o bem-estar relativo aos aspectos corporal, relacional, afetivo, cognitivo e espiritual, além do aspecto físico. O instrumento possui 5 respostas, que variam de 1 a 5 pontos, que vão entre corcordo totalmente a discordo totalmente JAMAS MT, 2013).

O instrumento BMSP 2 possui 7 domínios, distribuídos em 47 itens: Qualidade do relacionamento durante o cuidado (13 itens), Autocuidado e conforto (9 itens), Condições que propiciam o contato mãe e filho (4 itens), Cuidado despersonalizado (6 itens), Participação familiar contínua (4 itens), Cuidado oportuno e respeitoso (6 itens), Ambiente físico confortável (5 itens) (JAMAS MT, 2013).

Os dados deste estudo foram coletados a partir da Escala de Bem-Estar Materno em Situação de Parto BMSP2. A população deste estudo foi formada por mulheres no pós-parto após no mínimo, 8 horas do parto/nascimento, internadas no alojamento conjunto da maternidade Municipal. A coleta de amostras ocorreu entre outubro e novembro de 2021.

As pesquisadoras ficaram pelo período de 2 meses na instituição, incluindo finais de semana e períodos noturnos para realização da coleta de dados. As mesmas foram abordadas explicando as finalidades do estudo e sua importância. Inicialmente era preenchido o Termo de Consentimento Livre e Esclarecido (TCLE) junto as pesquisadoras, e posteriormente o questionário era entregues as pacientes, que é autoaplicável, onde tinham o tempo de 20 minutos para o preenchimento. Após assinatura uma via ficou com as pacientes e a outra via com as pesquisadoras.

Foram incluídas no estudo: mulheres maiores de 18 anos, puérperas de parto vaginal ou cesárea, de gestação única de termo maior ou igual a 37 semanas, ter parto realizado por médico ou enfermeiro; não ter apresentado qualquer complicação durante a gravidez/parto; ter permanecido no mínimo quatro horas no setor de pós-parto da instituição do estudo.

Foram excluídas: mulheres portadoras de alguma deficiência cognitiva, mulheres com distúrbios psiquiátricos e as com patologia exclusivamente ginecológica, abortamento, gestação ectópica, múltipla ou de feto pré-termo no momento da internação, bem como qualquer complicação grave ou por óbito fetal.

Para análise todos os dados do questionário foram repassados para uma planilha eletrônica em Excel padronizada, onde realizou-se a soma total dos pontos para os 47 itens, com relação a pontuação dos itens 5, 32, 33, 35 e 43, referentes ao domínio "cuidado despersonalizado", foi invertida antes da somatória. Através dessa planilha foi possível obter as seguintes variáveis: total de pontos por paciente, total de pontos por domínio por paciente e total, média, mediana e porcentagem referente a média.

Este estudo foi aprovado pelo Comitê de Ética em Pesquisa (CEP) da Universidade UNINORTE, com no de parecer 5.032.789.

\section{RESULTADOS}

Participaram deste estudo, 56 puérperas atendidas na maternidade municipal, que tiveram parto normal e cesárea. A análise descritiva do questionário foi realizada através da soma total das respostas obtidas para os 47 itens. Em cada um dos domínios, estão apresentadas as pontuações mínimas e máximas, sendo que quanto maior o valor alcançado mais positivo será a avaliação do bem-estar em relação à assistência ao parto.

A Tabela 1 está identificada pelas seguintes variáveis referente ao questionário completo e os sete domínios: nome dos domínios, número de itens, intervalo possível (mínimo e máximo), intervalo obtido (mínimo e máximo), mediana, média e porcentagem calculada conforme a média de pontos. 
O intervalo obtido no questionário $(n=56)$ foi de 121 a 234 pontos. Ainda em relação ao valor do total da versão adaptada da BMSP 2, foi constatada uma mediana de 204,5. Não se observa grande diferença entre os valores da mediana e da média (198) para o total da medida do bem-estar.

Por meio do escore total do instrumento, é possível explorar o bem-estar materno em três níveis: ótimo (pontuação > 200); adequado (pontuação entre 183 e 200) e mal-estar (pontuação < 183). Com relação ao total de pontos da escala, que foi 198 média e 204,5 mediana, o local de estudo classifica-se em adequado, por possuir uma pontuação entre 183 e 200.

A adaptação e tradução da escala do bem-estar em situação de parto, o ponto de corte relacionado a média de pontos, é de $77,8 \%$ do valor total possível, avaliados de forma individual. Ou seja, os domínios que estiverem acima de $77,8 \%$ são considerados resultados positivos, e os que estão abaixo são considerados resultados negativos (JAMAS MT, 2013).

Dos sete domínios avaliados, observou-se que em seis domínios, a porcentagem de pontos está acima de $77,8 \%$ do valor total possível, demonstrando um resultado positivo. O único domínio com valor abaixo do ponto de corte refere-se ao item "Cuidado despersonalizado", ficando com $72 \%$, conforme se observa na Tabela 1.

Tabela 1 - Estatística descritiva dos resultados total e por domínio da escala do bem-estar ( $n=56)$.

\begin{tabular}{lcccccc}
\hline \multicolumn{1}{c}{ Escala/Domínios } & $\begin{array}{c}\mathbf{N}^{\circ} \text { de } \\
\text { itens }\end{array}$ & $\begin{array}{c}\text { Intervalo } \\
\text { Possível }\end{array}$ & $\begin{array}{c}\text { Intervalo } \\
\text { Obtido }\end{array}$ & Mediana & Média & Porcentagem \\
\hline $\begin{array}{l}\text { Escala de Bem-Estar } \\
\text { Materno em Situação de }\end{array}$ & 47 & $47-235$ & $121-234$ & 204,5 & 198 & 84 \\
Parto & 13 & $13-65$ & $22-65$ & 58,5 & 57 & 88 \\
\hline $\begin{array}{l}1-\text { Qualidade do } \\
\text { relacionamento durante o } \\
\text { cuidado }\end{array}$ & 9 & $9-45$ & $16-45$ & 39 & 37 & 83 \\
\hline $\begin{array}{l}2-\text { Autocuidado e conforto } \\
\text { - Condições que } \\
\text { propiciam o contato mãe e }\end{array}$ & 4 & $4-20$ & $13-20$ & 19,5 & 19 & 93 \\
filho & 6 & $6-30$ & $12-30$ & 22 & 22 & 72 \\
\hline $\begin{array}{l}4-\text { Cuidado } \\
\text { despersonalizado }\end{array}$ & 4 & $4-20$ & $8-20$ & 17 & 17 & 83 \\
\hline $\begin{array}{l}5-\text { Participação familiar } \\
\text { contínua }\end{array}$ & 6 & $6-30$ & $12-30$ & 26,5 & 25,5 & 85 \\
\hline $\begin{array}{l}6-\text { Cuidado oportuno e } \\
\text { respeitoso }\end{array}$ & 5 & $5-25$ & $15-25$ & 22 & 22 & 87 \\
\hline $\begin{array}{l}7-\text { Ambiente físico } \\
\text { confortável }\end{array}$ & & & & \\
\hline
\end{tabular}

Fonte: Oliveira O, et al., 2021.

Na Tabela 2, estão apresentados os dados da classificação de bem-estar conforme os três níveis, de acordo com pontuação obtida de cada paciente. Das 56 mulheres que participaram deste estudo. Constatouse que $52 \%$, mais da metade das mulheres $(n=29)$ experimentaram ótimo bem-estar durante o parto, $16 \%$ das mulheres apresentaram nível adequado de bem-estar $(n=9)$ e $32 \%$ apresentaram mal-estar em relação à assistência ao parto $(n=18)$. De acordo com a média de pontos que foi obtida, o cenário do estudo foi classificado como bem-estar 'adequado' no parto, apresentando média de 198 pontos.

Tabela 2 - Classificação de bem-estar de acordo com pontuação obtida na escala $(n=56)$.

\begin{tabular}{lccc}
\hline Classificação & Pontuação & $\mathbf{N}^{\circ}$ & $\%$ \\
\hline Mal-estar & $<183$ & 18 & 32 \\
Adequado & $183>$ X $<200$ & 9 & 16 \\
Ótimo Bem-estar & $>200$ & 29 & 52 \\
\hline Total & & $\mathbf{5 6}$ & $\mathbf{1 0 0}$ \\
\hline
\end{tabular}

Fonte: Oliveira O, et al., 2021. 


\section{DISCUSSÃO}

A humanização envolve o acolhimento digno, tanto da mulher, como do bebê e da família, mediante condutas éticas e solidárias. Sendo assim, é necessário a organização de instituições para o parto, com ambiente e profissionais que estão em harmonia para alcançar o bem-estar no parto (MONTEIRO MS, et al., 2020).

O instrumento é dividido em sete domínios: qualidade do relacionamento durante o cuidado, autocuidado e conforto, condições que propiciam o contato mãe e filho, cuidado despersonalizado, participação familiar contínua, cuidado oportuno e respeitoso e ambiente físico e confortável (JAMAS, 2013). A discussão dos resultados deste estudo será norteada a partir dos domínios da Escala de Bem-Estar Materno em Situação de Parto.

\section{Qualidade do relacionamento durante o cuidado}

Com relação ao domínio que avalia a qualidade do relacionamento, o mesmo apresentou um resultado positivo, estando com $88 \%$ de aprovação com base no total de pontos, portanto, acima do ponto de corte. Tal domínio avalia questões de relacionamento por parte do profissional relacionado ao comportamento da mesma, como: tratamento respeitoso, cuidadoso, respeito as crenças e valores.

No momento do parto e pós-parto a equipe de saúde deve estar preparada para agir com a parturiente de uma forma centrada, tanto em técnicas e cuidados específicos, como na sua individualidade como ser humano, utilizando-se de uma abordagem empática, acolhedora e respeitosa, procurando ouvir suas demandas, queixas, inseguranças, medos, de forma que gere uma aproximação humanizada (ALVES BS e SANTOS MTB, 2020).

A assistência humanizada estabelece que os profissionais de saúde respeitem os aspectos fisiológicos do parto, e quando houver intervenção, que a mesma ocorra de forma cautelosa, reconhecendo os aspectos culturais e sociais da mulher, obtendo uma assistência com respeito, dignidade e autonomia (FREIRE LN e SILVA GSV, 2019).

\section{Autocuidado e conforto}

O autocuidado consiste em ações realizadas pelas puérperas para promoção particular da saúde e do bem-estar. E para efetivação dessas atividades de autocuidado, é necessária uma construção dialógica, que permite a troca de experiências, oferecendo uma assistência integral. Através da prática educativa oportuniza-se esses cuidados, prevenindo intercorrências, promovendo saúde e minimizando inseguranças e anseios, durante o pós-parto (BARBOSA EMG, et al., 2020).

O domínio que avalia o autocuidado e conforto trata-se do segundo domínio, o mesmo apresentou 83\% de aprovação com relação aos pontos totais. Estando acima do ponto de corte, que é de $77,8 \%$. Tal resultado demonstra que o local de estudo apresenta práticas educativas que proporcionam resultados positivos no bem-estar no parto.

Dentre alguns cuidados que podemos citar relacionados a autocuidado, temos higiene corporal, alimentação adequada, cuidado das mamas, relação sexual, repouso, saúde mental, cuidados pós cirúrgicos, orientações de aleitamento materno (SARTORI CC, et al., 2020).

\section{Condições que propiciam o contato mãe e filho}

O domínio que avalia as condições que propiciam o contato mãe e filho, apresentou a maior percentualidade, com $93 \%$ em relação aos pontos totais desse domínio. A pontuação mínima dessa categoria é de 4 pontos, podendo chegar a 20, os resultados deste estudo encontraram um intervalo de 13 pontos mínimo e 20 pontos no máximo, o que resultou em uma média de 19 pontos, contribuindo para o bom desempenho desse domínio.

Ao avaliar as práticas obstétricas hospitalares e suas repercussões no bem-estar materno em relação à assistência ao recém-nascido, Alvares AS, et al. (2019) encontrou resultados relacionando o bem-estar materno com o incentivo ao aleitamento materno, onde mães que foram incentivadas no aleitamento materno nos primeiros momentos do bebê tiveram mais chance de apresentar o bem-estar. 
O Contato Pele a Pele (CPP) entre a puérpera e o recém-nascido realizado ainda na sala de parto, na sua primeira hora de vida, é considerado ainda, além dos inúmeros benefícios, uma das ações de incentivo ao aleitamento materno, um aleitamento materno bem-sucedido tem relação com o primeiro contato realizado entre a mãe e o bebê (ABDALA LG e CUNHA MLC, 2018).

Além de estimular o vínculo da mãe e do bebê, o contato pele a pele traz diversos benefícios para o bebê, como proporcionar regulação de temperatura corporal, adaptação metabólica e redução de dor, mas também tal contato traz benefício a puérpera, pois através desse contato com o bebê, o corpo libera ocitocina, contração uterina e reduz o risco de hemorragia, além de aumentar vínculo da mãe e do bebê (JUNG SM; RODRIGUES FA e HERBER S, 2020).

O Ministério da Saúde possui um selo de qualidade, intitulado Iniciativa Hospital da Criança (IHAC), que é fornecido a instituições que cumprem critérios estabelecidos, para que possam ser consideradas uma instituição "Amigo da Criança". Dentre os critérios temos temas relacionados ao cuidado respeitoso e humanizado durante as fases do parto, garantia livre de acesso à mãe e pai e a permanência deles junto ao recém-nascido, cumprirem a Norma Brasileira de Comercialização para Lactentes e Crianças de Primeira Infância, Bicos, Chupetas e Mamadeiras e cumprimento dos dez passos do aleitamento materno (NBCAL) (LAMOUNIER JA, et al., 2019).

A maternidade municipal, cenário deste estudo, ganhou o selo no ano de 2019 por cumprir os critérios que são estabelecidos, além disso, o estabelecimento se mantém como o único hospital do Estado que conta com esse título.

Os dados achados neste estudo vão ao encontro do título que é mantido pela instituição, que é de proporcionar um bem-estar materno com diversos critérios exigidos, o que corrobora com os domínios avaliados através do questionário que foi aplicado.

\section{Cuidado despersonalizado}

O domínio que avalia o cuidado despersonalizado, apresentou a menor porcentagem, estando inclusive abaixo do score que foi definido como critério. Com relação a esse domínio, o mesmo faz os seguintes questionamentos: senti que alguns atendimentos foram bruscamente realizados por membro da equipe? me senti tratada de forma autoritária? "foram mandões comigo?"; sinto que foram realizados procedimentos que não correspondem ao processo de um parto natural?; muitas vezes evitei expressar a dor?, meu mal-estar ou desconfortos ou inconveniência, por medo de que as pessoas da equipe de saúde se indispusessem comigo?; senti que me atenderam em um antigo hospital e em más condições físicas e houve momentos em que eu me senti abandonada, como se as minhas necessidades fossem indiferentes para a equipe?. Este domínio faz questionamentos relacionados ao relacionamento interpessoal estabelecido pelos profissionais, estratégias de alívio dos desconfortos do parto e à alimentação.

A pontuação mínima desse domínio é de 6 pontos e no máximo 30, este trabalho encontrou um intervalo de 12 a 30 pontos, o que resultou em $72 \%$ com relação aos pontos totais desse domínio. Conforme observado, das 56 pacientes, das seis perguntas deste domínio, a pergunta que apresentou o número menor de pontos refere-se ao "Senti que alguns atendimentos foram bruscamente realizados por membro da equipe.

Muitos avanços aconteceram no processo de parto, e isso envolve avanços científicos e tecnológicos, porem essa assistência baseada apenas em tecnologia se torna repetitiva e mecânica, ocorrendo por muitas vezes de forma isolada e desumanizada, através de uso excessivo de intervenções, tais fatores favorecem por parte das parturientes, sentimentos de medo, ansiedade e insegurança, relacionada a equipe e local que é realizado tal momento (ROCHA NFF e FERREIRA J, 2020).

Para Oliveira, et al. (2017) mesmo com o avanço do programa de humanização do pré-natal e nascimento (PHPN), ainda ocorrem casos de mulheres que não estão sendo protagonistas do seu parto, não sendo reconhecidas na sua liberdade de escolha, do que seria melhor para seu conforto.

Valadão CM e Pegoraro RF (2020) demonstraram em seu estudo que as respectivas ideias sobre parto e vivência da mulher, que o momento de parir não é tão fácil, mas que, apesar de tudo, é muito especial para 
a vida de cada mulher. Observou-se a importância da humanização da equipe de saúde para tornar esse momento mais especial ainda. É visível que o momento do parto não é tão confortável, cabendo, à equipe de saúde do centro obstétrico, proporcionar conforto a essas mulheres.

Tendo em vista a baixa pontuação obtida no aspecto de cuidado despersonalizado, tornou-se evidente a necessidade de medidas para promover a qualidade da atenção quanto a este aspecto.

\section{Participação familiar contínua}

O domínio buscou analisar os dados relacionados ao direito e respeito de ter um acompanhante durante o processo do parto e pós-parto, ao verificar os dados desse domínio, o mesmo apresentou ótima avaliação, estando com $83 \%$ com base no valor total desse domínio. O que representa que o local de estudo não só estabeleceu, mas cumpre os requisitos relacionados a lei de ter um acompanhante, mas que também respeita.

Em 2000, ocorreu uma campanha nacional incentivando o direito à mulher de ter um acompanhante ao seu lado durante o trabalho de parto, respeitando as indicações da Organização Mundial de Saúde (OMS). Como resultado desse movimento, em 07 de abril de 2005 foi aprovada a Lei oㅜ 11.108, que garante à parturiente a escolha de um acompanhante durante o trabalho de parto, parto e pós-parto imediato nas maternidades conveniadas ao Sistema Único de Saúde (SUS). Tal acompanhante tem um papel importante, como suporte físico e emocional à mulher, age como um aliado, pois reflete as necessidades da parturiente e ajuda na observação da mesma e de seu estado (ALMEIDA NMO e RAMOS EMB, 2020).

Rosa SG, et al. (2020) ao analisar a percepção da gestante sobre o acompanhante durante o cenário do parto, observou que que a presença do acompanhante no decorrer do trabalho de parto, parto e pós-parto, trouxe um maior conforto, segurança e força durante todos os momentos, o que o torna parte que merece destaque ao lado da puérpera. Como relata a fala encontrada "É fundamental a gente ter esse apoio numa hora tão mágica, tão importante na vida de uma mulher, ele tá do lado dá mais segurança".

\section{Cuidado oportuno e respeitoso}

O domínio oportuno e respeitoso avalia questões relacionadas a privacidade, anestesia, informações de medicação, saúde do bebê e da mãe (JAMAS MT, 2013). Tal domínio apresentou ótima avaliação durante análise dos dados deste estudo, estando com $85 \%$ de aprovação com base na pontuação total. Demonstrando que a unidade, através dos profissionais apresentaram uma boa avaliação.

Esses achados vão ao encontro das recomendações acerca de como deve ser estabelecida a relação do profissional com a puérpera, contempladas nas Diretrizes nacionais de assistência ao parto normal, onde os profissionais de saúde devem respeitar as mulheres em trabalho de parto e estabelecer uma relação igualitária, sempre lhes perguntando sobre seus desejos e expectativas (TORRES RR, 2020).

No trabalho de parto é comum observar fatores como dor, sofrimento, solidão, angústia, onde toda a situação vivenciada amedronta a paciente, o que resulta em uma falta de controle das situações. Neste momento os profissionais de saúde são essenciais, fornecendo informações sobre a mãe e o bebê, evolução do parto, medicações ou outras, o que ajuda a reduzir os riscos de ter um parto traumatizante, bem como a probabilidade de ter complicações (NASCIMENTO FCV; SILVA MP e VIANA MRP, 2018).

\section{Ambiente físico confortável}

O domínio ambiente físico confortável avalia questões relacionadas a limpeza, iluminação, ambiente acolhedor e aparelhos adequados. Tal domínio apresentou ótima avaliação durante este estudo, estando com $87 \%$ de aprovação com base na pontuação total. Demonstrando que a unidade, através dos profissionais que realizam a limpeza e também de sua estrutura estão adequados conforme os quesitos que foram citados.

Corroborando com a Rede Cegonha através da portaria no 1.459, de 24 de junho 2011, onde a mesma consiste em uma rede de cuidados, visando: "assegurar à mulher o direito ao planejamento reprodutivo e à atenção humanizada à gravidez, ao parto e ao puerpério, bem como à criança o direito ao nascimento seguro e ao crescimento e ao desenvolvimento saudáveis", em seu item II, letra b a mesma cita a ambiência das maternidades como um componente essencial e necessário ao parto e nascimento humanizado. 


\section{CONCLUSÃO}

O estudo apresentou dados que possibilitaram a reflexão a respeito da assistência prestada durante o parto e pós-parto, visando a prática da humanização inserida nesses cuidados e de que forma tais cuidados afetam o bem-estar da mulher como protagonista.

Os resultados obtidos, mostram que o bem-estar materno está presente na unidade estudada, apesar do ponto relacionado ao relacionamento interpessoal estabelecido pelos profissionais, estratégias de alívio dos desconfortos do parto e à alimentação necessitarem de melhorias e investimentos, porém, a conscientização por parte dos profissionais de saúde dos benefícios trazidos por esse cuidado, têm se potencializado cada vez mais, com perspectivas futuras positivas quanto ao bem-estar da puérpera.

Como limitações para este estudo, refere-se a análise sob a perspectiva da mulher sobre seu atendimento. Torna-se relevante a análise a partir de outros olhares como por exemplo, dos profissionais de saúde e sua visão de cuidado inserido na assistência durante o parto.

Os resultados deste estudo evidenciam que políticas públicas bem conduzidas podem transformar o cenário da atenção ao parto e nascimento, promovendo o bem-estar materno em situação de parto, implicando diretamente na qualidade do cuidado ao binômio mãe e filho.

\section{REFERÊNCIAS}

1. ABDALA LG, CUNHA MLC. Contato pele a pele entre mãe e recém-nascido e amamentação na primeira hora de vida. Clin Biomed Revista, 2018; 38(4): 356-60.

2. ALMEIDA NMO, et al. O direito da parturiente ao acompanhante como instrumento de prevenção à violência obstétrica., 2020; 9(4): 12-27.

3. ALVARES AS, et al. Hospital obstetric practices and their repercussions on maternal welfare. Rev Esc Enferm USP. 2020; 54: e03606.

4. ALVES BS, SANTOS MTB. Humanização da assistência de enfermagem no parto. J Health Sci Inst, [S. I.], p. 1-5.

5. BARBOSA EMG, et al. Desenvolvimento e validação de cartilha educativa para saúde e bem-estar no pósparto*. Revista Rene, [S. I.], p. 1-7, 25 nov. 2021.

6. CAMPOS VS, et al. Experiência de puérperas com a dor do parto normal. Revista Eletrônica Acervo Saúde, 2020; Sup.40: 2396.

7. FERREIRA RC, et al. Práticas integrativas e complementares na assistência do período puerperal. Revista Eletrônica Acervo saúde, 2021; 13(1): e 5254.

8. FREIRE LN, SILVA GSV. A falta do protagonismo da mulher no trabalho de parto, parto e nascimento. Revista PróUniverSUS, 2019; 10(1): 34-37.

9. JAMAS MT. Adaptação cultural e validação para a língua portuguesa da "Escala de bienestar Materno em Situacion de Parto (BMSP 2)". Tese (Doutorado em enfermagem) - Universidade de São Paulo (USP), São Paulo, 2013; 152p.

10. JUNG SM, et al. Contato pele a pele e aleitamento materno: Experiências de Puérperas. Revista de Enfermagem do Centro Oeste Mineiro, 2020; 10: e3657.

11. LAMOUNIER JA, et al. Iniciativa Hospital Amigo Da Criança: 25 Anos De Experiência No Brasil. Revista Paulista de Pediatria, 2019; 37(4): 486-493.

12. LIMA WM, et al. A experiência do Parto a Partir do Olhar de Mães Adolescentes. Educação, Psicologia e Interfaces, 2018; 2: 121-134.

13. MONTEIRO MS, et al. Importância da assistência de enfermagem no parto humanizado. ReBIS Revista Brasileira Interdisciplinar de Saúde, 2020; 2(4): 1-8.

14. NASCIMENTO FCV, et al. Assistência de enfermagem no parto humanizado. Revista Pre Infec e Saúde, $2018 ; 4: 6$.

15. PEREIRA RM, et al. Novas práticas de atenção ao parto e os desafios para a humanização da assistência nas regiões sul e sudeste do Brasil. Ciência \& Saúde Coletiva [online]. 2018, 23(11).

16. RIBEIRO JF, et al. A. Assistência ao Parto Normal sob o Olhar da Parturiente. Revista Eletrônica Gestão \& Saúde, 2016; 7(1): 113-25.

17. ROCHA NFF, FERREIRA J. A escolha da via de parto e a autonomia das mulheres no Brasil: uma revisão integrativa. Saúde em Debate [online]. 2020; 44(125).

18. ROSA SG, et al. A presença do acompanhante no trabalho de parto, parto e pós-parto: compreensão das gestantes. Revista Pró-UniverSUS. 2020; 11(1): 21-26.

19. SARTORI CC, et al. AS CRENÇAS QUE INFLUENCIAM O AUTOCUIDADO DA PUÉRPERA. Brazilian Journal of Surgery and Clinical Research, [S. I.], p. 1-5.

20. STEEN M, FRANCISCO AA. Bem-estar e saúde mental materna. Acta Paulista de Enfermagem, 2019; 32(4): 3-6.

21. TORAL A, et al. Assistência de enfermagem na humanização do parto: uma revisão integrativa. Estácio Saúde, 2019 ; $8(1): 45-53$.

22. TORRES RS. Cuidados de Enfermagem no Parto e Nascimento. Braz. J. of Develop., [S. I.], p. 1-14.

23. VALADÃO CL, et al. Vivências de mulheres sobre o parto. Fractal: Revista de Psicologia [online]. 2020, 32(1): 91-98. 